\title{
Heparin-Induced Fever in Neurointensive Care Unit: A Rarity Yet a Possibility
}

\author{
Mathangi Krishnakumar ${ }^{1, \odot ~ S h w e t a ~ S . ~ N a i k ~}{ }^{1} \quad$ Venkatapura J. Ramesh ${ }^{1} \quad$ Mouleeswaran S1
}

${ }^{1}$ Department of Neuroanaesthesia and Neurocritical Care, National Institute of Mental Health and Neurosciences, Bengaluru, Karnataka, India

\begin{abstract}
Address for correspondence Mathangi Krishnakumar, MBBS, MD, PDF, DM, Department of Neuroanaesthesia and Neurocritical Care, National Institute of Mental Health and Neurosciences, Bengaluru, Karnataka 560030, India (e-mail: mathz89@gmail.com).
\end{abstract}

\begin{abstract}
Keywords

- heparin

- drug fever

- hyperthermia

- NICU

- neurological disease
\end{abstract}

\section{Introduction}

Fever is the most common symptom in neurointensive care unit (NICU), seen in almost $70 \%$ of the patients. ${ }^{1} \mathrm{~A}$ rise in core temperature has detrimental effects on the already injured brain in terms of increasing brain metabolic requirement, oxygen consumption, and can further promote cardiopulmonary injury. ${ }^{2}$ In NICU, refractory and recurrent fever without an identifiable infectious source is common. Most of these fevers are treated with antibiotics adding to cost and emergence of resistant strains. ${ }^{3,4}$

Fever contributes to increased duration of stay, worsened outcomes, and higher mortality in NICU; therefore, it is imperative to identify the cause and treat fever at the earliest. ${ }^{5}$ Fever due to drug reaction is often overlooked and contributes to 2 to $3 \%$ of cases in intensive care unit (ICU); incidence in NICU is not known. ${ }^{6}$ The incidence increases with usage of more drugs and longer duration. Drugs that have been implicated in causing fevers include various anticonvulsants, allopurinol, and certain antibiotics with nitrofurantoin, minocycline, and sulfonamides being the most common. ${ }^{7}$ Heparin has been documented as causing drug-induced fever (DIF) with only two previously reported cases by Forni and Murray and Laun et al., 8

published online

November 27, 2021
DOI https://doi.org/ $10.1055 / \mathrm{s}-0041-1739350$ ISSN 2348-0548

\section{Case Report}

A 32-year-old female with Guillain-Barré syndrome on ventilator had an episode of bradycardia with asystole on day 2 of NICU admission. Return of spontaneous circulation was achieved in less than 5 minutes, and neurological examination after 72 hours showed decline in cognitive function with preserved consciousness. She was able to follow verbal commands. Magnetic resonance imaging brain revealed hypoxic changes in the cerebellum and occipital cortex. She underwent five cycles of plasmapheresis, was tracheostomized on day 12 , and continued to be on mechanical ventilation. She had no other comorbidities. She was diagnosed with deep vein thrombosis of right popliteal vein on day 13 on routine ultrasound screening. She was treated with subcutaneous heparin 10,000 IU followed by dose-adjusted heparin to maintain activated prothrombin time $>1.5$ times and transitioned to oral warfarin on day 18 . She developed bleeding from trachea; hence, warfarin was stopped and she was restarted on heparin 5,000 IU every 12 hours. On day 21 , she was noted to have fever ranging from 101 to $103.6^{\circ} \mathrm{F}$. The fever was intermittent without touching baseline. She was managed initially on lines of infection and empirically treated with antibiotics. To determine the source of infection tracheal culture, urine

(c) 2021. Indian Society of Neuroanaesthesiology and Critical Care. All rights reserved.

This is an open access article published by Thieme under the terms of the Creative Commons Attribution-NonDerivative-NonCommercial-License, permitting copying and reproduction so long as the original work is given appropriate credit. Contents may not be used for commercial purposes, or adapted, remixed, transformed or built upon. (https://creativecommons.org/licenses/by-nc-nd/4.0/).

Thieme Medical and Scientific Publishers Pvt. Ltd. A-12, 2nd Floor, Sector 2, Noida-201301 UP, India 
culture and blood cultures were done. All reports over next 2 weeks remained negative. She underwent multiple chest X-ray, deep venous thrombosis screen, ultrasonography of abdomen, renal system, and echocardiography to look for vegetation and signs of infective endocarditis, all remained negative. Thyroid function tests, autoimmune profile, rheumatological panel, tests for fungal, mycobacteria, viral infections like hepatitis, human immunodeficiency virus, herpes simplex virus, cytomegalovirus, rabies, and Japanese encephalitis were unremarkable. Procalcitonin done at all-time points over 2 weeks remained $<0.1 \mathrm{ng} / \mathrm{mL}$. Renal function tests, liver function tests, lipid profile, erythrocyte sedimentation rate, and peripheral smear for parasites were normal during the period of febrile episodes.

Drug fever reaction and central fever were considered as a differential diagnosis as physical examination and investigations did not reveal any abnormality. Medications, which were suspected to be causative, were stopped in stepwise manner, simultaneously looking for change in her fever pattern (-Fig. 1). However, fever pattern continued; at this stage the medications were reconsidered. After careful review of literature and her medication, heparin was considered the probable cause for her fever. On day 31, heparin treatment was discontinued, and after 20 hours, the patient's temperature was less than $100^{\circ} \mathrm{F}$. She remained afebrile and asymptomatic for the next 3 days. There were no fever spikes 24 to $48 \mathrm{~h}$ after stopping heparin (-Fig. 2). Heparin was replaced with low molecular weight heparin; she remained afebrile the following weeks. On day 72 of ICU admission, she was weaned off ventilator and shifted to ward with no further complications.

\section{Discussion}

The general dictum in the management of fever of unknown origin in critical care is that "Therapy should be withheld, whenever possible, until the cause of fever has been determined, so that it can be tailored to a specific diagnosis." ${ }^{10}$ In the NICU, the clinician combats inherent limitations of the physical exam and neurological exam, while racing against the incubation period for cultures, and keeping in mind the critical sequelae of untreated infections or unmanaged central neurogenic fever. Regardless, the adverse effects of temperature elevation on neurological recovery are clear.

Clinical presentation of patients with infectious and noninfectious has considerable overlap. ${ }^{3}$ Our patient had multitude of possibilities as etiology for fever-thromboembolism, nosocomial infection, and central fever. A stepwise approach was followed to rule out infectious cause of fever. A central cause could not be completely ruled out in this case as the patient had neurological sequelae following cardiac arrest. The onset of fever following drug initiation, nonspecific pattern, resistance to conventional treatment, and extensive testing ultimately led to considering DIF as the possible etiology.

The incidence of DIF is unknown and is usually a diagnosis of exclusion. The mechanisms described for DIF include effect of drug on thermoregulation, pharmacokinetics, administration related effect, idiosyncratic reaction, and hypersensitivity reaction. The drugs that have been implicated are described in previous studies. ${ }^{7}$ Discontinuing all medications at once may put the patient at risk from the cessation of the drug as well as prevent identification of the causative agent. The timing of onset is variable, on an average 7 to 10 days; fever is cyclical and resolves 72 to 96 hours after cessation of the offending drug. ${ }^{6,7}$ Our patient exhibited many of the above-stated findings, negative infectious workup as well as laboratory abnormalities, and onset after 8 days that all eventually improved after discontinuation of the heparin. The resolution of fever was seen in less than 24 hours. The previous reported heparin-induced fever has been in a burn patient and in a patient with inferior vena cava thrombosis following pelvic inflammatory disease. In these patients became afebrile within 24 hours and 8 hours respectively. ${ }^{9}$

Our case demonstrates what is most likely a heparin-induced fever in a neurologically ill patient after ruling out the other possible etiologies. To the best of our knowledge, this is the first reported case in an NICU setup.

\section{Conclusion}

In NICU, it is imperative to have a pragmatic approach to diagnosis of fever to prevent secondary brain injury. DIF needs reasonable consideration when other etiologies do not fit the clinical or laboratory picture.

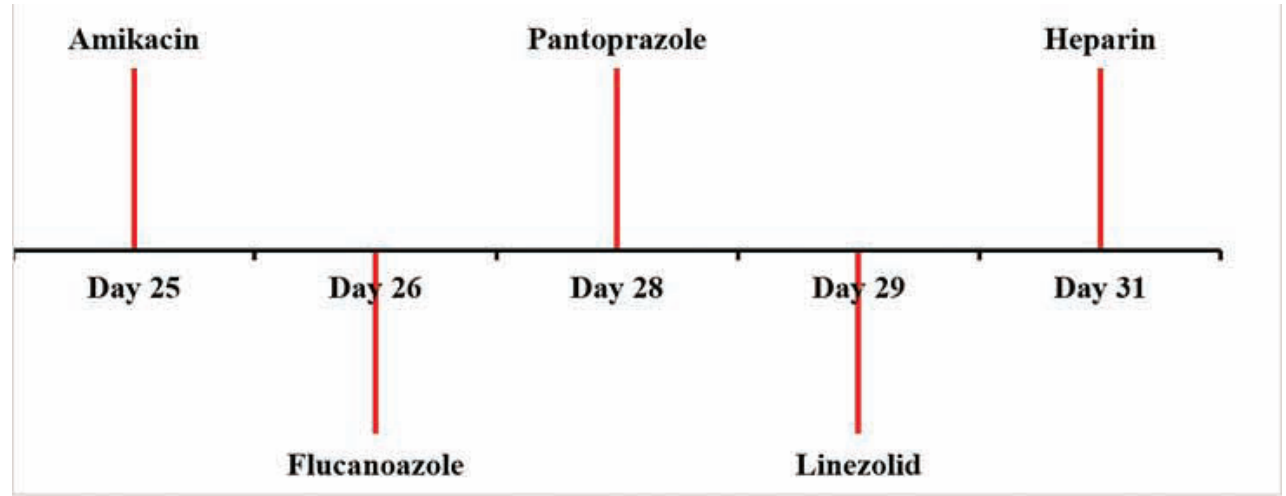

Fig. 1 Stepwise discontinuation of drugs. 


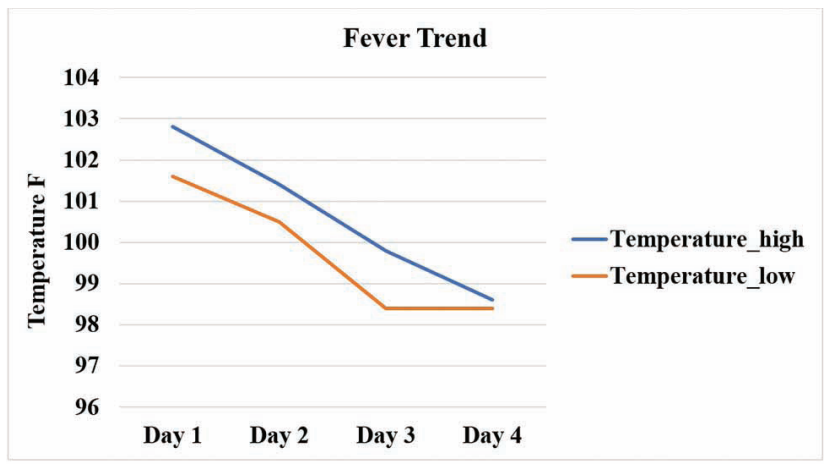

Fig. 2 Fever pattern after stopping heparin.

\section{Conflict of Interest}

None declared.

\section{References}

1 Badjatia N. Fever control in the neuro-ICU: why, who, and when? Curr Opin Crit Care 2009;15(2):79-82
2 Lopez GA. Temperature management in the neurointensive care unit. Curr Treat Options Neurol 2016;18(3):12

3 Rabinstein AA, Sandhu K. Non-infectious fever in the neurological intensive care unit: incidence, causes and predictors. J Neurol Neurosurg Psychiatry 2007;78(11):1278-1280

4 Commichau C, Scarmeas N, Mayer SA. Risk factors for fever in the neurologic intensive care unit. Neurology 2003;60(5):837-841

5 Meier K, Lee K. Neurogenic fever. J Intensive Care Med 2017;32(2):124-129

6 Roush MK, Nelson KM. Understanding drug-induced febrile reactions. Am Pharm 1993;NS33(10):39-42

7 Patel RA, Gallagher JC. Drug fever. Pharmacotherapy 2010; 30(1):57-69

8 Forni AL, Murray HW. Drug fever induced by heparin. Am J Med 1992;92(1):107

9 Laun J, Laun K, Farooqi A, Smith DJ. Heparin-induced fever: a case report and literature review. J Burn Care Res 2019;40(5):723-724

10 Laws C, Jallo J. Fever and infection in the neurosurgical intensive care unit. JHN Journal 2010;5(2):23-27 